Pre-print of: Ali Hasanabadi, Majid Baniassadi, Karen Abrinia, Masoud Safdari \& Hamid Garmestani (2016). 3D microstructural reconstruction of heterogeneous materials from 2D cross sections: A modified phase-recovery algorithm. Computational Materials Science. DOI: 10.1016/j.commatsci.2015.09.015

\title{
3D Microstructural Reconstruction of Heterogeneous Materials from 2D Cross Sections: A Modified Phase-Recovery Algorithm
}

\author{
Ali Hasanabadi ${ }^{1}$, Majid Baniassadi ${ }^{1,2}$, Karen Abrinia ${ }^{1}$, Masoud Safdari ${ }^{3 *}$ and Hamid Garmestani ${ }^{4}$ \\ ${ }^{1}$ School of Mechanical Engineering, College of Engineering, University of Tehran, P.O. Box 111554563, Tehran, \\ Iran \\ ${ }^{2}$ University of Strasbourg, ICube/CNRS, 2 Rue Boussingault, 67000 Strasbourg, France \\ ${ }^{3}$ Aerospace Engineering Department, University of Illinois, 104 S Wright St., Urbana, Illinois 61801, USA \\ ${ }^{4}$ School of Materials Science and Engineering, Georgia Institute of Technology, United States
}

\begin{abstract}
A novel method for the 3D reconstruction of a microstructure from limited statistical information provided by 2D cross sections is developed. In the proposed approach, first full-set statistical information (two-point correlation functions) are extracted from 2D cross sections, and then an approximate 3D microstructure is realized based on them. The proposed method relies mainly on conditional probability theorem to establish explicit functional forms between two-point correlation functions extracted from 2D cross sections and full-set 3D statistics. For 3D realization, a novel phase-recovery algorithm is developed that captures prominent attributions of the microstructure. The salient feature of the proposed realization scheme is the ability to fully reconstruct 3D microstructures from statistical information provided by just one cross section for isotropic microstructures and two perpendicular cross sections for anisotropic ones. A number of illustrative examples are provided to demonstrate the accuracy and the versatility of the proposed scheme. The application of the method for the 3D realization of microstructure using an experimental dataset is demonstrated. Finally, the accuracy of the method in capturing and retaining essential features including volume fractions and characteristic attributions as well as the state of anisotropy and percolation of the phases is discussed.
\end{abstract}

Keywords: Reconstruction; 2-point statistics; approximate correlations; phase recovery

\section{Introduction}

Key performance characteristics of the heterogeneous materials is often directly related to their microstructure. A comprehensive understanding about the link between the properties and the microstructural features of these materials may require a full 3D picture of the microstructure (e.g. to estimate transmission properties). However, usually for applications in material science, petroleum engineering, biology and medicine, only 2D image(s) are available. Different reconstruction techniques are developed to realize 3D microstructures from the 2D images based on the material system of interest and available imaging modalities. For applications in materials science, a subsequent analysis step often follows the 3D reconstruction step to obtain desired macroscopic properties (e.g., mechanical, transport, and electromagnetic properties) [1-4] .

Many studies show that some of the effective properties of a random heterogeneous material are strongly correlated with a particular formalism called n-point statistics $[5,6]$. N-point correlation functions can be exploited directly to characterize effective mechanical, thermal, electrical and permeability properties of a wide range of heterogeneous material systems $[1,5,7]$. Through these functions, characteristic statistics of a microstructure are translated into a set of distributions that systematically provide more information about the microstructure with increasing their order. In other word, every microstructure can be uniquely reconstructed from infinite-order correlation functions [8-10].

\footnotetext{
${ }^{*}$ Corresponding author, Tel/Fax: 505-550-9792, e-mail: $\underline{\text { msafdari@ @illinois.edu }}$
} 
Pre-print of: Ali Hasanabadi, Majid Baniassadi, Karen Abrinia, Masoud Safdari \& Hamid Garmestani (2016). 3D microstructural reconstruction of heterogeneous materials from 2D cross sections: A modified phase-recovery algorithm. Computational Materials Science. DOI: 10.1016/j.commatsci.2015.09.015

Traditionally, statistical information extracted from experimental measurements and morphological assessments are used to quantify microstructural attributions [11]. Direct reconstruction of heterogeneous microstructures practiced by stitching digitized serial section images acquired by FIB-SEM, X-ray computed tomography (microCT), scanning laser confocal microscopy and other imaging modalities are not well-suited to routine engineering applications due to expensive technology, lack of skilled operators and many other technical issues involved $[12,13]$. The need for reliable alternative computational methods for the 3D microstructural reconstruction from limited statistical information have been raised lately in different disciplines related to the materials research as successful reconstruction procedures can provide nondestructive and low-cost means of estimating the macroscopic properties of a heterogeneous materials with complex internal microstructure.

3D microstructural reconstruction from limited microstructural information obtained from $2 \mathrm{D}$ cross sections is a classic inverse problem with many engineering applications in efficient design of material, statistical analysis and visualization. Historically Gaussian filtering methods are extensively used for this purpose $[14,15]$. These methods usually rely on two-point probability functions and apply linear/nonlinear filters on Gaussian random fields to match the correlation function in during reconstruction step. Lanzini et al. [16] used truncated-Gaussian method for 3D reconstruction from 2D phase images. Aside from their considerable computational cost, this method and similar ones are only applicable for two-phase isotropic media.

Using a suitable descriptor of initial 2D slices, optimization-based approaches are also developed for the 3D reconstruction purposes $[17,18]$. Yeong and Torquato developed a stochastic optimization technique to generate microstructure from a specified set of statistical two-point correlation functions [17]. In their study, a two-dimensional slice of the microstructure is utilized to obtain morphological information and perform full 3D realization. Similarly, Bochenek and Pyrz used simulated annealing procedure to reconstruct spatial dispersions of inclusions in a media guided by observation from 2D images. In their approach, they used field quantities like interface stresses as additional constraints in the optimization procedure to achieve a better realization [18]. Sundararaghavan and Zabaras utilized a pattern recognition technique to reconstruct microstructures [19]. In this method, support vector machines are implemented to generate a class of microstructures with close statistical characteristics to the real microstructure and to develop a reconstruction procedure based on a hierarchical classification scheme.

More recent studies include a novel Monte Carlo methodology to reconstruct 3D microstructures of multi-phase heterogeneous materials from a 2D SEM micrograph developed by Baniassadi et al. [20]. In order to perform reconstruction from a single digitized cross section image, they developed a hybrid stochastic technique for realizing microstructure from two-point correlation functions that employs colony and kinetic growth algorithms for the realization step and provides a coupled realization-optimization methodology.

In the current study, a novel computational framework is developed for the 3D reconstruction of microstructure from 2D cross sections of statistically homogenous isotropic and anisotropic microstructures. An explicit formulation is developed to approximate two-point correlation functions of a 3D microstructure. In this method, 3D two-point correlation functions are first approximated from statistical information for a given set of orthogonal planes. To deal with issues arising from ill-posed nature of the problem, we resort to conditional probability theorem to provide enough constraints needed to achieve an optimum closed-form approximation. The approximate formulation is the extension of the recent work by Baniassadi and co-workers were N-point correlation functions of heterogeneous materials are investigated [21, 22]. In the next step, microstructure reconstruction is performed by employing a novel phase recovery algorithm which takes as input approximate two-point correlation functions developed in the initial step. It should be mentioned that phase recovery algorithms has been deployed for many other applications including signal processing [23]. Initially proposed by Fullwood and co-workers [7, 24, 25], these algorithms are used for the reconstruction of a microstructure from its two-point correlation functions. Contrasting to more traditional methods based on simulated annealing, phase recovery algorithms can be utilized to reconstruct 3D multiphase microstructures 
Pre-print of: Ali Hasanabadi, Majid Baniassadi, Karen Abrinia, Masoud Safdari \& Hamid Garmestani (2016). 3D microstructural reconstruction of heterogeneous materials from 2D cross sections: A modified phase-recovery algorithm. Computational Materials Science. DOI: 10.1016/j.commatsci.2015.09.015

from large databases, which hinders standard computational approaches [24]. Current study summarizes with a series of experimental and numerical case studies to illustrate the computational efficiency and capabilities of the proposed approach.

\section{Two-Point Probability Functions}

\subsection{Basic Concepts}

Statistical correlation functions can be utilized for mathematical description of the morphology of microstructure. For any n-phase random heterogeneous medium, the characteristic function for one of the phases (say phase i) is defined as

$$
\chi^{(\text {phase } i)}(x)=\left\{\begin{array}{l}
1 \\
0 \quad \text { otherwise }
\end{array}\right.
$$

where $\mathrm{x}$ represents the position vector of a point in the heterogeneous microstructure. If we consider $\mathrm{N}$ points within the microstructure randomly positioned at $x_{1}, x_{2}, \ldots, x_{N}$, the probability of the event in which all these point belong to the phases $\mathrm{i}=1 \ldots \mathrm{n}$ is defined as $\mathrm{N}$-point correlation function (Fig. 1). The $\mathrm{N}$-point correlation functions are then defined by

$$
C_{N}^{i j \ldots n}\left(x_{1}^{i}, x_{2}^{j}, \ldots, x_{N}^{n}\right)=\left\langle\chi^{i}\left(x_{1}\right), \chi^{j}\left(x_{2}\right), \ldots, \chi^{n}\left(x_{N}\right)\right\rangle, \quad i, j, \ldots, n \in \text { set of phases },
$$

where $\langle\ldots\rangle$ is the ensemble average.

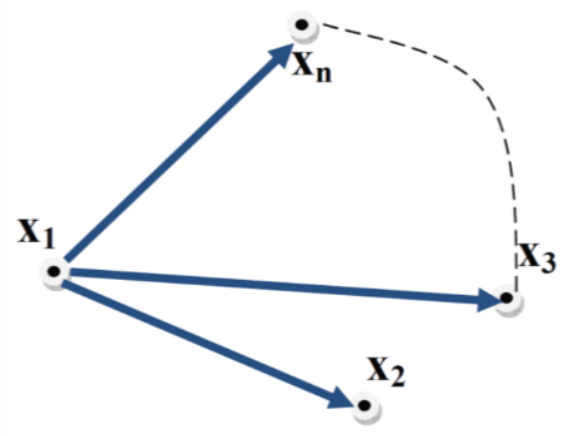

Fig. 1. Schematic of correlation vectors of the n-point correlation functions

For different value of N, N-point correlation functions cover different level of information about the microstructure they represent. For instance, one-point correlation functions capture only the volume fraction of each phase for multiphase materials or the orientation distribution function (ODF) for polycrystalline materials [1]. The constraint on the one-point correlations can be expressed as

$$
\sum_{i=1}^{n} C_{1}^{i}\left(x_{1}^{i}\right)=1 .
$$

Similarly, two-point correlation functions describe the joint probability distribution of finding starting and ending points of a predefined vector within specific states. This collective information are conveyed through two-point correlation functions, measured by all possible vectors relevant to the microstructure. In general, for a d-dimensional isotropic media, two-point correlation functions can be extracted from an $\mathrm{m}$-dimensional subspace $(\mathrm{m}=1,2 \ldots \mathrm{d}-1)$. For 
Pre-print of: Ali Hasanabadi, Majid Baniassadi, Karen Abrinia, Masoud Safdari \& Hamid Garmestani (2016). 3D microstructural reconstruction of heterogeneous materials from 2D cross sections: A modified phase-recovery algorithm. Computational Materials Science. DOI: 10.1016/j.commatsci.2015.09.015

example, two-point correlation functions of a $3 \mathrm{~d}$ isotropic microstructure can be extracted from a $2 \mathrm{~d}$ or $1 \mathrm{~d}$ cross section of it [26]. The two-point correlations of eigen microstructures can be calculated using the square of the amplitude of the Fast Fourier Transform (FFT) of the characteristic function. Higher-order correlation functions can be computed also using higher-order spectra [7]. Generally, an eigen microstructure is defined such that in each grid point there is only one local state. All non-eigen microstructures are considered as eigen microstructures when the grid is sufficiently fine [24].

Let us denote the head and tail points of vector $(\vec{r})$ by $x_{1}$ and $x_{2}$, and define $C_{2}^{i j}\left(x_{1}^{i}, x_{2}^{j}\right)$ as follow:

$C_{2}^{i j}\left(x_{1}^{i}, x_{2}^{j}\right)=P\left\{\left(x_{1} \in \varphi_{i}\right) \cap\left(x_{2} \in \varphi_{j}\right)\right\}=C_{2}^{i j}\left(x_{1}^{i}, x_{2}^{j}\right),\left(x_{1} \in \varphi_{i}\right) \equiv x_{1}^{i}, i, j=1,2$.

Using Eq. (4), for N-point correlation functions we get

$$
P\left\{\left(x_{1} \in \varphi_{i}\right) \cap\left(x_{2} \in \varphi_{j}\right) \cap \ldots \cap\left(x_{N} \in \varphi_{n}\right)\right\}=C_{N}^{i j \ldots n}\left(x_{1}^{i}, x_{2}^{j}, \ldots, x_{N}^{n}\right) .
$$

Due to normality conditions the following constraints are satisfied [27]:

$$
\begin{gathered}
\sum_{i=1}^{n} \sum_{j=1}^{n} C_{2}^{i j}\left(x_{1}^{i}, x_{2}^{j}\right)=1 \quad \text { where } \vec{r}=x_{2}^{j}-x_{1}^{i}, \\
\left.\sum_{\substack{j=1 \\
C_{2}^{i j}}}^{n} C_{2}^{i j}\left(x_{1}^{i}, x_{2}^{j}\right)=x_{2}^{j}\right)=v_{i}^{j i}\left(x_{2}^{j}, x_{1}^{i}\right)
\end{gathered}
$$

where $v_{i}$ is volume fraction of phase i. In addition, the following well-known limits exist for the values of the twopoint correlation functions as $\vec{r}$ approaches zero and infinity [27]:

$$
\begin{aligned}
& \lim _{x_{2}^{j} \rightarrow x_{1}^{i}} C_{2}^{i j}\left(x_{1}^{i}, x_{2}^{j}\right)=\left\{\begin{array}{ll}
v_{i} & \text { if } i=j \\
0 & \text { if } i \neq j
\end{array},\right. \\
& \lim _{\left(x_{2}^{j}-x_{1}^{i}\right) \rightarrow \infty} C_{2}^{i j}\left(x_{1}^{i}, x_{2}^{j}\right)=\left\{\begin{array}{cc}
v_{i}^{2} & \text { if } i=j \\
v_{i} v_{j} & \text { if } i \neq j
\end{array} .\right.
\end{aligned}
$$

Using conditional probability, correlation functions can be defined based on other probability functions as follows:

$$
\begin{gathered}
C_{2}^{i j}\left(x_{1}^{i}, x_{2}^{j}\right)=P\left\{\left(x_{2} \in \varphi_{j}\right) \mid\left(x_{1} \in \varphi_{i}\right)\right\} P\left(x_{1} \in \varphi_{i}\right), \\
C_{3}^{i j k}\left(x_{1}^{i}, x_{2}^{j}, x_{3}^{k}\right)=P\left\{\left(x_{3} \in \varphi_{k}\right) \mid\left(x_{1} \in \varphi_{i}\right) \cap\left(x_{2} \in \varphi_{j}\right)\right\} P\left\{\left(x_{2} \in \varphi_{j}\right) \mid\left(x_{1} \in \varphi_{i}\right)\right\} P\left(x_{1} \in \varphi_{i}\right),
\end{gathered}
$$

\subsection{Approximation}

In the present study, 2-point correlation functions of 3D microstructures are needed to be approximated for all grid points (vectors) of eigen microstructure from two-point correlation of 2D images. For simplicity, two phase microstructures are utilized to extract TPCFs and implementing 3D-reconstruction approach, however, the proposed method can be extended to multiphase microstructure.

To calculate two-point correlation function of a supposed statistical vector $\vec{r}$, it is decomposed into two perpendicular vectors $\vec{r}_{X Y}$ and $\vec{r}_{Z}$ as shown in Fig. 2. 
Pre-print of: Ali Hasanabadi, Majid Baniassadi, Karen Abrinia, Masoud Safdari \& Hamid Garmestani (2016). 3D microstructural reconstruction of heterogeneous materials from 2D cross sections: A modified phase-recovery algorithm. Computational Materials Science. DOI: 10.1016/j.commatsci.2015.09.015

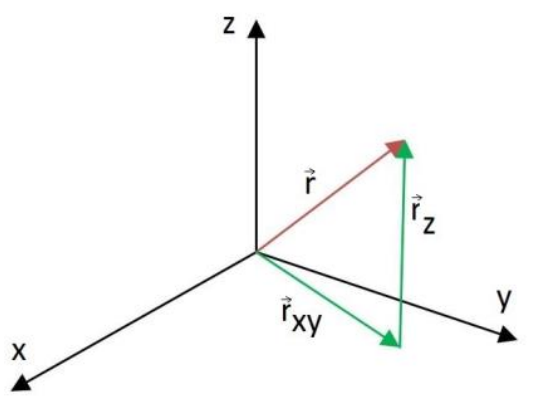

Fig. 2. Decomposition of the vector $\vec{r}$ into two perpendicular vectors $\vec{r}_{X Y}$ and $\vec{r}_{Z}$

We consider $x_{1}$ as the starting point of vector $\vec{r}$ and $\vec{r}_{X Y}, x_{3}$ as the end point of the vector $\vec{r}$ and $\vec{r}_{Z}$, and $x_{2}$ as the end point of vector $\vec{r}_{X Y}$ and starting point of $\vec{r}_{Z}$. The TPCF for the vector $\vec{r}$ with its head and tail residing in i-phase and k-phase, respectively, can be calculated using the sum of the probability of two mutually exclusive events as follows [28],

$$
\begin{gathered}
C_{2}^{i k}\left(x_{1}^{i}, x_{3}^{k}\right)=P\left\{\left(x_{1}^{i}, x_{2}^{i}, x_{3}^{k}\right) \cup\left(x_{1}^{i}, x_{2}^{j}, x_{3}^{k}\right)\right\}=P\left\{\left(x_{1}^{i}, x_{2}^{i}, x_{3}^{k}\right)\right\}+P\left\{\left(x_{1}^{i}, x_{2}^{j}, x_{3}^{k}\right)\right\} \\
=C_{3}^{i i k}\left(x_{1}^{i}, x_{2}^{i}, x_{3}^{k}\right)+C_{3}^{i j k}\left(x_{1}^{i}, x_{2}^{j}, x_{3}^{k}\right) . i, j, k \in\{1,2\}
\end{gathered}
$$

Based on Eq. (13), in order to calculate TPCF, we should calculate 3-point correlation functions. Recently, Baniassadi et al. proposed a method to formulate a theoretical approximation for the full set of N-point correlation function based on subsets of (N-1)-point correlation function, a set of weight functions, conditional probability (Eqs. (11), (11)), and boundary conditions (Eqs. (8), (9)) [21, 22].

In this approximation, weight functions are used to connect subsets of (N-1)-point correlation functions for estimating the full set of N-point correlation function (Eq. (14)).

Considering a composite with two phases $\varphi_{1}$ and $\varphi_{2}$ the proposed approximation states:

$$
\begin{gathered}
C_{3}^{i j k}\left(x_{1}^{i}, x_{2}^{j}, x_{3}^{k}\right) \approx W_{1} \frac{C_{2}^{i k}\left(x_{1}^{i}, x_{3}^{k}\right) C_{2}^{i j}\left(x_{1}^{i}, x_{2}^{j}\right)}{v_{i}}+W_{2} \frac{C_{2}^{i k}\left(x_{2}^{i}, x_{3}^{k}\right) C_{2}^{i j}\left(x_{1}^{i}, x_{2}^{j}\right)}{v_{j}}+W_{3} \frac{C_{2}^{i k}\left(x_{1}^{i}, x_{3}^{k}\right) C_{2}^{j k}\left(x_{2}^{j}, x_{3}^{k}\right)}{v_{k}} \\
i, j, k \in\{1,2\},(14)
\end{gathered}
$$

where $\mathrm{W}_{1}, \mathrm{~W}_{2}$ and $\mathrm{W}_{3}$ are the dependency weight functions.

In the proposed approximation, a unique solution does not exist for the weight functions. Therefore, any chosen set of the weight functions that satisfy the necessary boundary limit conditions is useful for this approximation. For example, for the approximation of three-point correlation functions, the weight functions can be expressed as:

$$
W_{p}=\frac{\left|x_{q} x_{r}\right|^{\alpha}}{\left|x_{q} x_{r}\right|^{\alpha}+\left|x_{p} x_{q}\right|^{\beta}+\left|x_{p} x_{r}\right|^{\gamma}},
$$

where $\mathrm{p}, \mathrm{q}$ and $\mathrm{r}$ are equal to 1,2 or 3 and $\alpha, \beta$ and $\gamma$ are non-zero positive real numbers [22]. Optimum values of parameters $\alpha, \beta$ and $\gamma$ can be calculated using an optimization scheme. Properties of weight functions have been discussed in detail in the referenced articles [21, 22]. One of these properties that is used in developing of approximation is:

$$
W_{1}+W_{2}+W_{3}=1 .
$$


Pre-print of: Ali Hasanabadi, Majid Baniassadi, Karen Abrinia, Masoud Safdari \& Hamid Garmestani (2016). 3D microstructural reconstruction of heterogeneous materials from 2D cross sections: A modified phase-recovery algorithm. Computational Materials Science. DOI: 10.1016/j.commatsci.2015.09.015

Using Eqs. (6)-(8), (14) and (15) it is possible to estimate $C_{3}^{i i k}\left(x_{1}^{i}, x_{2}^{i}, x_{3}^{k}\right)$ and $C_{3}^{i j k}\left(x_{1}^{i}, x_{2}^{j}, x_{3}^{k}\right)$ according to relevant TPCFs. Under these conditions and using Eqs. (13) and (16), the weight functions are eliminated, and we finally obtain

$$
C_{2}^{i k}\left(x_{1}^{i}, x_{3}^{k}\right) \approx \frac{C_{2}^{i i}\left(x_{1}^{i}, x_{2}^{i}\right) C_{2}^{i k}\left(x_{2}^{i}, x_{3}^{k}\right)}{v_{i}}+\frac{C_{2}^{i j}\left(x_{1}^{i}, x_{2}^{j}\right) C_{2}^{j k}\left(x_{2}^{j}, x_{3}^{k}\right)}{v_{j}}
$$

Due to the elimination of the weight functions, the obtained solution will be unique, and there is no concern about optimization of weight functions (Baniassadi et al. [22], proposed optimization of $\alpha, \beta$ and $\gamma$ for better approximation).

For n-phase microstructure, the solution can be generalized as follows;

$$
C_{2}^{i k}\left(x_{1}^{i}, x_{3}^{k}\right) \approx \sum_{j=1}^{n} \frac{C_{2}^{i j}\left(x_{1}^{i}, x_{2}^{j}\right) C_{2}^{j k}\left(x_{2}^{j}, x_{3}^{k}\right)}{v_{j}}
$$

For example for a 2-phase heterogeneous material, using Eq. (17), $C_{2}^{11}\left(x_{1}^{1}, x_{3}^{1}\right)$ is defined by

$$
C_{2}^{11}\left(x_{1}^{1}, x_{3}^{1}\right) \approx \frac{C_{2}^{11}\left(x_{1}^{1}, x_{2}^{1}\right) C_{2}^{11}\left(x_{2}^{1}, x_{3}^{1}\right)}{v_{1}}+\frac{C_{2}^{12}\left(x_{1}^{1}, x_{2}^{2}\right) C_{2}^{21}\left(x_{2}^{2}, x_{3}^{1}\right)}{v_{2}} .
$$

\section{Microstructure Reconstruction}

Reconstruction process is the realization of a microstructure from its specific statistical information. In this research, microstructure reconstruction has been performed using approximate two-point correlation functions of a 3D microstructure. Several numerical methods have been proposed to reconstruct microstructure using statistical information [1, 19, 29-31]. In most of reconstruction techniques, an optimization approach is usually employed to minimize statistical error between a proposed trial microstructure and a target microstructure; therefore, these methods are best described as optimization problems. Generally, gradient-based schemes can be used to find a solution to such optimization problems, and these methods converge faster than other techniques in cases they are applicable [29]. One of the pitfalls of gradient-based approaches is their poor performance in finding global optima in problems with several local optima [29]. Simulated annealing is a capable optimization reconstruction technique that is highly useful for problems with many local optima. This technique is commonly used in the optimization of statistical functions[1]. Despite these appealing properties mentioned above, it should be noted that the simulated annealing or gradient methods are computationally demanding approaches, and therefore they are not feasible techniques for the reconstruction purposes with current state computing power, especially for the multiphase microstructures [24].In this research, phase-recovery algorithms are exploited to reconstruct microstructure following Fullwood et al. [24]. Phase recovery algorithms are also mostly used for signal processing application, however we show that these algorithms can be useful for reconstruction purposes using limited statistical information as well. Compared to mentioned methods, phase recovery method has lower computational cost for multi-phase microstructures. This advantage and other properties such as consistency with spectral representation methods have motivated us to use it for microstructural reconstruction. We target eigen microstructures for which a 3D regular grid is used to digitize and represent them and each grid point is only occupied by one phase.

This dataset or microstructure state is denoted here by $m_{s}^{n}$, where the superscript $n$ enumerates the phase number and the subscript $s$ enumerates number of each grid which is defined the microstructure. Microstructure state, $m_{s}^{n}$, is the existence probability for phase $\mathrm{n}$ in the position $\mathrm{s}$; therefore, it is zero or one for eigen microstructures. This condition is mathematically described by 
Pre-print of: Ali Hasanabadi, Majid Baniassadi, Karen Abrinia, Masoud Safdari \& Hamid Garmestani (2016). 3D microstructural reconstruction of heterogeneous materials from 2D cross sections: A modified phase-recovery algorithm. Computational Materials Science. DOI: 10.1016/j.commatsci.2015.09.015

$$
\sum_{n=1}^{N} m_{s}^{n}=1, \quad m_{s}^{n} \in\{0,1\}
$$

where $\mathrm{N}$ denotes the number of phases in the microstructure, and $\mathrm{S}$ is the total number of grid points of the microstructure. It is noted this definition for microstructure function, $m_{s}^{n}$, is identical with characteristic function defined in Eq. (1). Therefore one-point correlation functions can be defined simply by

$$
C_{1}^{n}=\frac{1}{S} \sum_{s=0}^{S-1} m_{s}^{n}
$$

Similarly, discretized two-point correlation functions are defined using

$$
C_{2}^{n n^{\prime}}(r)=\frac{1}{S} \sum_{s=0}^{S-1} m_{s}^{n} m_{s+r}^{n^{\prime}}
$$

where the superscripts $n$ and $n^{\prime}$ denote the phases of interest and the subscript $r$ enumerates discretized correlation vectors which can be used to describe the statistics of microstructure. Using fast Fourier transform (FFT) for the microstructure function, we get

$$
M_{k}^{n}=\mathcal{F}\left(m_{S}^{n}\right)=\frac{1}{S} \sum_{s=0}^{S-1} m_{S}^{n} \mathrm{e}^{2 \pi i s k} / s=\frac{1}{S}\left|M_{k}^{n}\right| \mathrm{e}^{i \theta_{k}^{n}},
$$

where $\left|M_{k}^{n}\right|$ is the amplitude, and $\theta_{k}^{n}$ is the phase of the Fourier transform. By applying convolution theorem to the FFT of Eq. (22), and by assuming periodicity of the structure, we obtain

$$
F_{k}^{n n^{\prime}}=\mathcal{F}\left(C_{2}^{n n^{\prime}}(r)\right)=\frac{1}{S}\left|M_{k}^{n}\right| \mathrm{e}^{-i \theta_{k}^{n}}\left|M_{k}^{n^{\prime}}\right| \mathrm{e}^{i \theta_{k}^{n^{\prime}}} .
$$

It is important to note that the FFT of any autocorrelation $\left(n=n^{\prime}\right)$ is the square of the amplitude of the FFT of the respective microstructure function without phase information defined by

$$
F_{k}^{n n}=\frac{1}{S}\left|M_{k}^{n}\right|^{2}=\frac{1}{S} \widetilde{M}_{k}^{n} * M_{k}^{n}
$$

where $\widetilde{M}_{k}^{n}$ is the complex conjugate of $M_{k}^{n}$. Eq. (23) and (25) can be employed for the reconstruction procedure, and generally approaches based on these relationships are known as phase-recovery algorithms. In the current study, twopoint correlation functions for the 3D microstructure are first approximated using Eq. (19), then we utilize phaserecovery reconstruction algorithms to acquire $m_{s}^{n}$ for reconstruction of the microstructure.

Phase recovery algorithm that is used for reconstruction, follows the Gerchberg-Saxton algorithm [23], and has four steps: (1) an initial random microstructure is guessed and the FFT of the microstructure function, $m_{s}^{n}$, is calculated using Eq. (23); (2) only the modulus of the guess is replaced with the square root of the approximated autocorrelation multiplied by S (Eq. (25) and the phases remain unchanged; (3) inverse Fourier transform is calculated (using inverse of Eq. (23)); and finally (4) the constraints in real space (Eq. (20)) are imposed. The generated realization acquired through described phase-recovery algorithm is then used in the step 1 as initial microstructure, and the iterations continue till satisfaction of a specific criteria. In the current approach, a desirable error between the two-point correlation functions of initial and target microstructure is adopted as stopping criteria.

When phase, $\theta_{k}^{n}$, for state $\mathrm{n}$ is recovered, using Eq. (24), it is possible to obtain $\theta_{k}^{n^{\prime}}$ for other state $n^{\prime}$. Therefore using inverse Fourier transform of Eq. (23), $m_{s}^{n^{\prime}}$ is obtained.

To compare the computation speed of phase recovery algorithm and other methods, simulated annealing method that is used frequently in reconstruction process, was selected [1, 30, 31]. For a 200x200 cell structure it lasted $2400 \mathrm{~s}$ to achieve error equal to $10^{-8}$ where it lasted only $15 \mathrm{~s}$ for phase recovery method for the same microstructure size, error of reconstruction (Fig. 9) and processor. It is notable that this microstructure was isotropic and only the 
Pre-print of: Ali Hasanabadi, Majid Baniassadi, Karen Abrinia, Masoud Safdari \& Hamid Garmestani (2016). 3D microstructural reconstruction of heterogeneous materials from 2D cross sections: A modified phase-recovery algorithm. Computational Materials Science. DOI: 10.1016/j.commatsci.2015.09.015

magnitude of TPCFs were considered. Reconstruction of anisotropic and multiphase microstructure is impossible using traditional computing power. Moreover this method is very sensitive to considered parameters such as initial temperature, temperature decreasing strategy and so on [32].

\section{Results and Discussion}

\subsection{D Approximation}

First, we present a simple hypothetical 2-D microstructure to illustrate the procedure of approximation. In this case, we approximated TPCFs of all grid points using only TPCFs of X and Y directions. Fig. 3a depicts a two-phase microstructure, where one of the phases is colored black and the other is white. This image was taken from Jiao work [30]. He has generated this image by the lattice-point algorithm and a damped-oscillating correlation function. The microstructure information was stored on a regular 200x200 square grid. The volume fraction of the white phase in this microstructure was $v_{1}=0.34$. The complete set of 2-point statistics of white phase were calculated using Eq. (25) and 2-point statistics in $\mathrm{X}$ and $\mathrm{Y}$ directions were extracted. Then the complete set of 2-point statistics of all grid points for white phase were approximated using Eqs. (6)- (8), (19) and correlation functions of only $\mathrm{x}$ and y directions.

Using approximated TPCFs and phase-recovery algorithms, reconstruction of microstructure was done (Fig. 3b,c). Comparison of results reveals that reconstructed microstructure using approximate TPCFs has retained its original features such as geometric connectivity and percolation of phases. It should be noted that the reconstructed microstructure is only used 400 arrays of total 40000 arrays of reconstruction matrix (about $1 \%$ ).

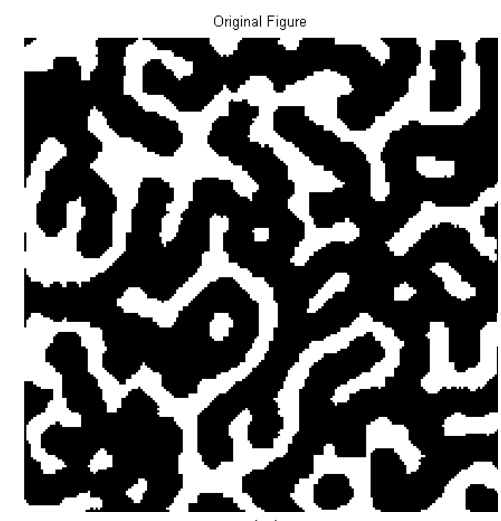

(a)

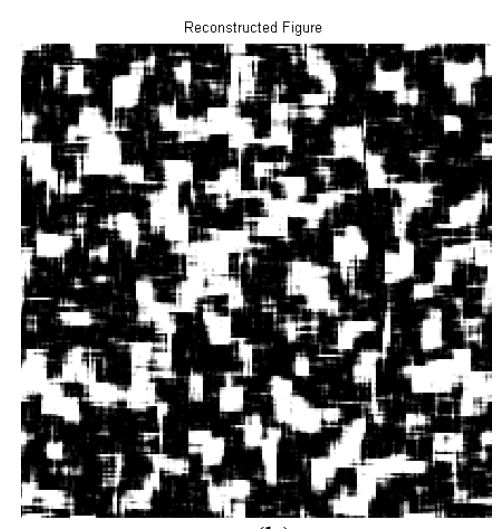

(b)

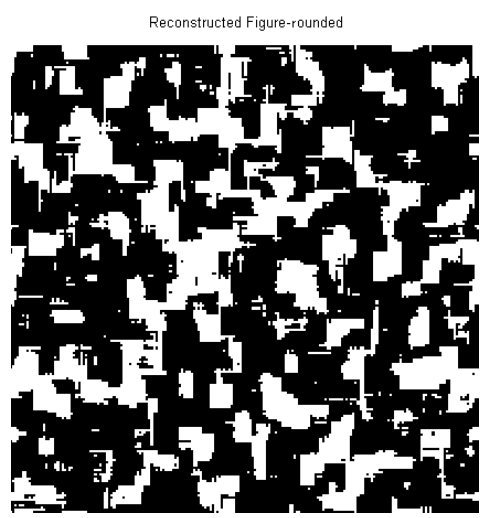

(c)

Fig. 3. Reconstruction of 2D microstructure; (a) original microstructure, (b) reconstructed figure and (c) removing gray pixels of reconstructed image by rounding off fractional pixels.

\subsection{Isotropic Materials}

In this case, we generated three-dimensional statistically homogeneous and isotropic media using statistical information of the two-dimensional section described in 4.1 (Fig. 3a). This section was a 200x200 structure with black and white phases, and we generated a 3-D 200×200x200 (8 million cells) structure using proposed approximation and reconstruction method.

Because the structure was statistically homogeneous, we used identical TPCFs for both xy and yz-plane (Fig. 2). Using Eq. (19) and (25), we calculated full set of autocorrelation functions of white phase and the reconstructed 3-D 
Pre-print of: Ali Hasanabadi, Majid Baniassadi, Karen Abrinia, Masoud Safdari \& Hamid Garmestani (2016). 3D microstructural reconstruction of heterogeneous materials from 2D cross sections: A modified phase-recovery algorithm. Computational Materials Science. DOI: 10.1016/j.commatsci.2015.09.015

microstructure using prescribed phase-recovery algorithm. Using Eqs. (6)-(8), it is clear that in a two- phase microstructure, the full 2-point functions is uniquely defined by the autocorrelation function of either of phases.

The 3D reconstructed microstructure is shown in Fig. 4. The cross sections corresponding to $x=1,80,150$ and 200 pixel are shown, respectively. Comparison of original section (Fig. 3a) and these sections reveal that original features of microstructure such as connectivity and smoothness are retained slightly better than 2D reconstruction (Fig. 3c).

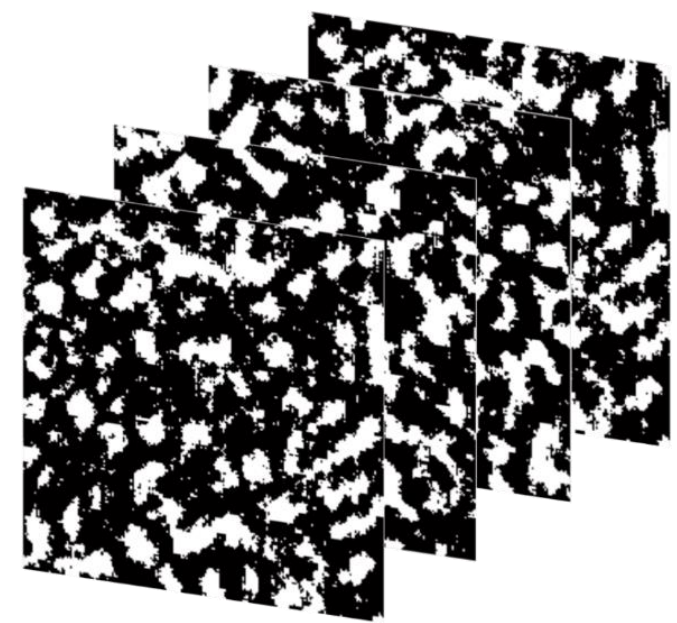

Fig. 4. 3D reconstructed microstructure, sections are shown on $\mathrm{x}=1,80,150$ and 200 pixel, from front to back, respectively.

It should be noted that because we approximated correlation functions, we should not expect that reconstructed sections exactly resemble the original section. But coherency and connectivity of structure are features that are retained properly as shown in Fig. 5a-d for $x=99-102$ pixel, respectively.

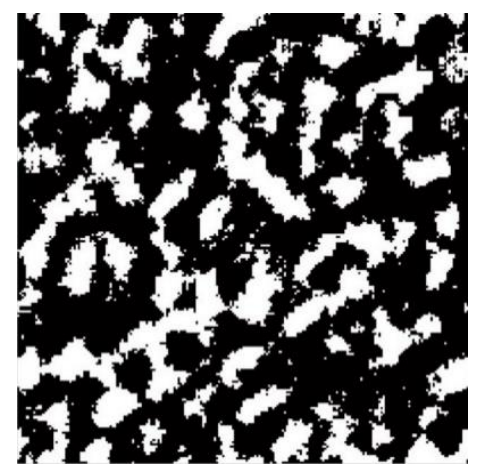

(a)

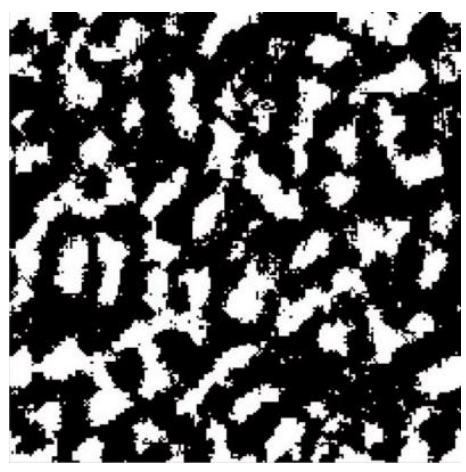

(b)

\subsection{Anisotropic Materials}

Metallographers often examine two mutually perpendicular sections to study and quantify anisotropic microstructures (for example, longitudinal and transverse planes of cold-rolled plate). In order to capture all features of anisotropic microstructure, we used two cross sections perpendicular to the $\mathrm{x}$ - and z-axis (Fig. 6(a) and 6(b), respectively).Original $3 \mathrm{D}$ microstructure is a $150 \times 150 \times 150$ structure with two phases black $\left(v_{1}=0.59\right)$ and white $\left(v_{1}=0.41\right)$. Simulation 
Pre-print of: Ali Hasanabadi, Majid Baniassadi, Karen Abrinia, Masoud Safdari \& Hamid Garmestani (2016). 3D microstructural reconstruction of heterogeneous materials from 2D cross sections: A modified phase-recovery algorithm. Computational Materials Science. DOI: 10.1016/j.commatsci.2015.09.015

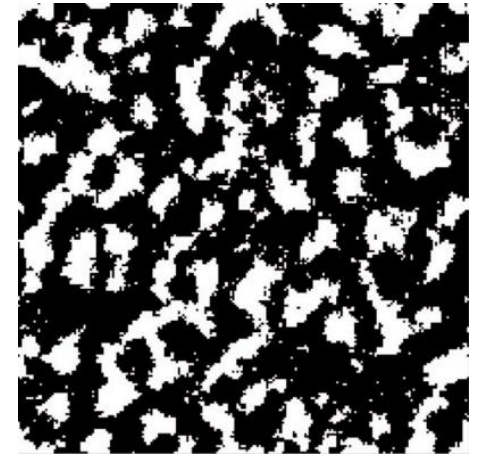

(c)

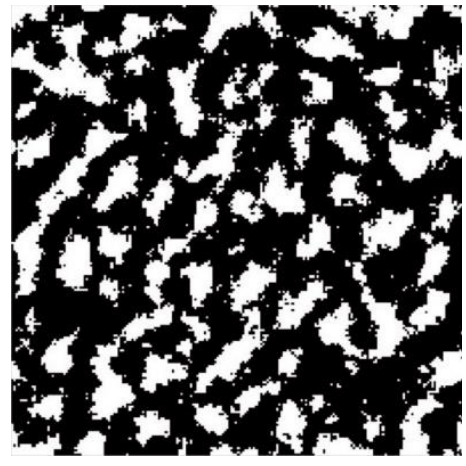

(d)

Fig. 5. Percolation and connectivity of phases that are retained properly, (a)-(d) are $\mathrm{x}=99-102$ pixel, respectively.

of the original microstructure is carried out by a hybrid stochastic methodology based on the colony and kinetic algorithms and Monte Carlo methodology [20].

The reconstructed 3D anisotropic microstructure is shown in Fig.6 and the original and approximated TPCFs in the diagonal direction are depicted in Fig 7. The periodicity assumption for the structure (Eq. (24)), enforces the autocorrelation functions to start from volume fraction of white phase $\left(v_{1}=0.41\right)$, approach to $v_{1}^{2}(=0.17)$, and then increase to a value close to $v_{1}$.

Comparison of Fig. 6a,b and 3D reconstructed microstructure (Fig. 6c-e) reveals that anisotropy of microstructure is retained properly (for example anisotropy of white phase in diagonal direction).

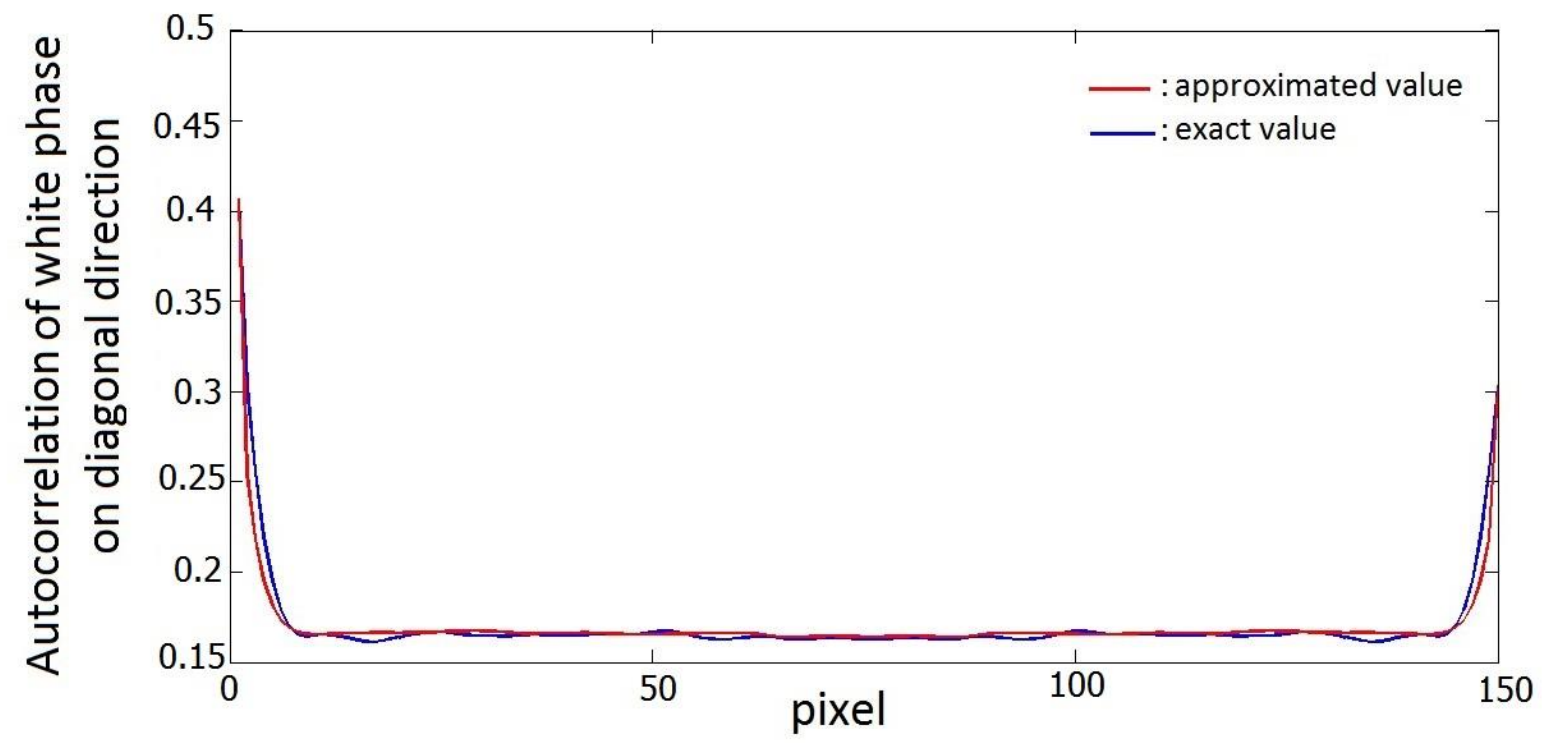

Fig. 7. Exact (blue) and approximated (red) autocorrelation functions of white phase in diagonal direction 
Pre-print of: Ali Hasanabadi, Majid Baniassadi, Karen Abrinia, Masoud Safdari \& Hamid Garmestani (2016). 3D microstructural reconstruction of heterogeneous materials from 2D cross sections: A modified phase-recovery algorithm_Somnutational Materials Science. DOI: 10.1016/i.commatsci.2015.09.015

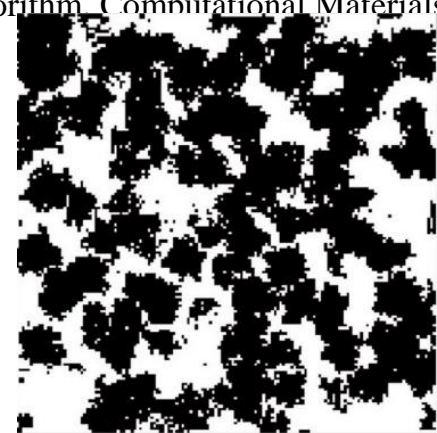

(a)

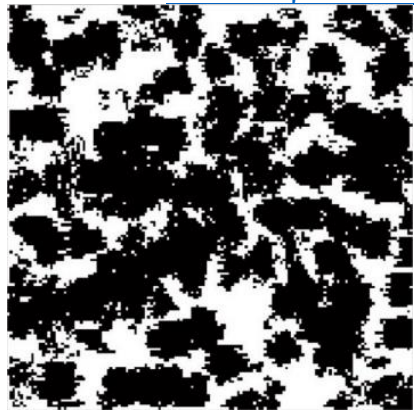

(b)

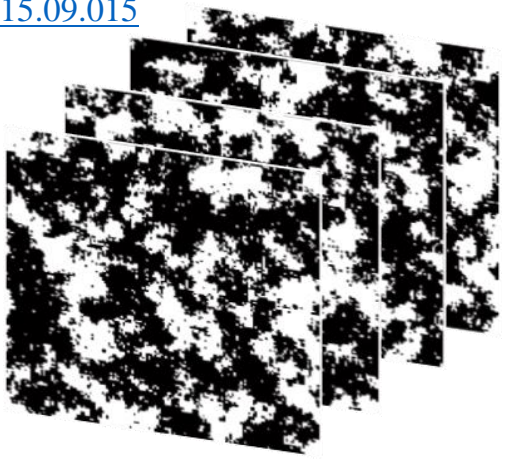

(c)

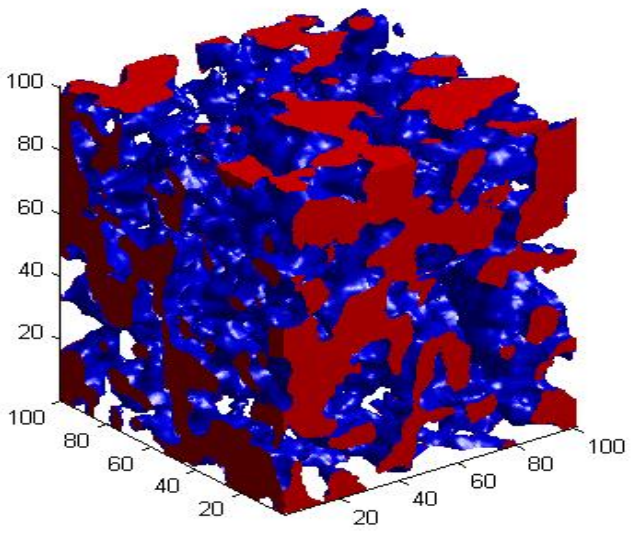

(d)

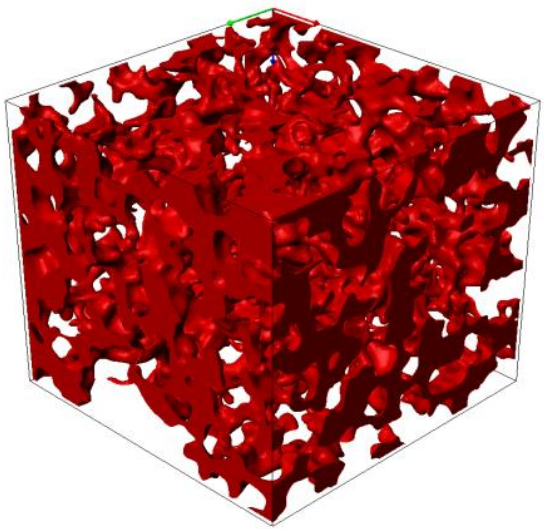

(e)

Fig. 6. Two cross sections perpendicular to the (a) $\mathrm{x}$ - and (b) z-axis. (c) 3D reconstructed media, sections are shown on $\mathrm{z}=1,50,100$ and 150 pixel, from front to back, respectively. (d) 3-D reconstruction of the microstructure based on the presented methodology. (e) 3-D reconstruction of the microstructure based on serial sectioning.

\subsection{Experimental Investigation}

To evaluate the capability of the proposed method in practical applications, 3-D reconstruction of a Halloysite nanotube (HNT) polypropylene composite, using one cross section was performed. This cross section was obtained using focused ion beam (FIB), and scanning electron microscopy (SEM) [13]. The microstructure cross section was a regular 300 x 300 square grid and the volume fraction of filler of the composite or white phase (HNT) was 0.11 (Fig. $8 a)$.

Comparison of 3-D reconstructed microstructure, shown in Fig. 8b, with original cross sections of microstructure, shown in Fig. 8a, demonstrates that the most essential features of original nano-composite such as size, shape, and distribution of phases are retained using the proposed method. Comparison of volume fraction of initial cross section (0.1098) and obtained 3-D microstructure (0.1151) revealed the acceptable error of reconstruction (less than 5\%).

Baniassadi et al. [13], used the FIB-SEM method for serial sectioning of the nano-composite and created many slices. By stacking these slices and a 3-D maker software they obtained 3-D microstructure of the nano-composite (Fig. 8c). But in proposed method, using only one cross section, we calculated TPCFs and obtained essential features of the microstructure with a faster and less expensive method (Fig. 8d). 
Pre-print of: Ali Hasanabadi, Majid Baniassadi, Karen Abrinia, Masoud Safdari \& Hamid Garmestani (2016). 3D microstructural reconstruction of heterogeneous materials from 2D cross sections: A modified phase-recovery algorithm. Computational Materials Science. DOI: 10.1016/j.commatsci.2015.09.015

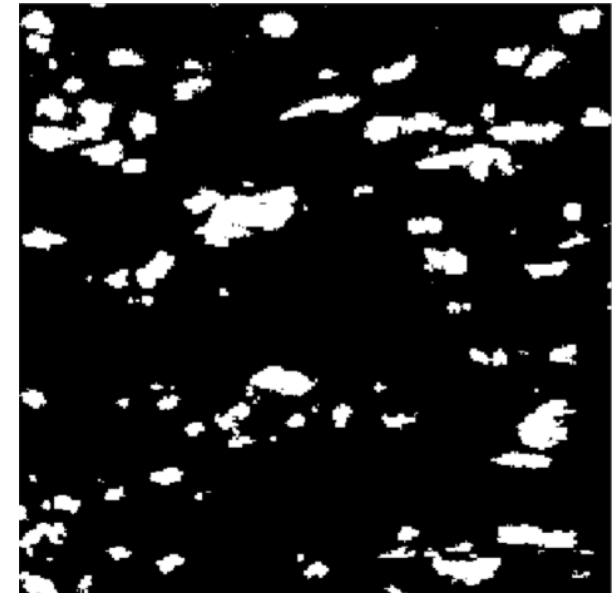

(a)

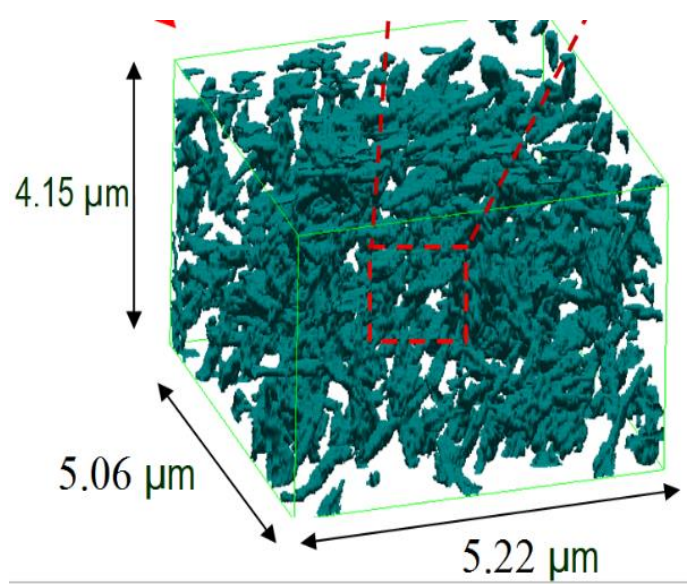

(c)

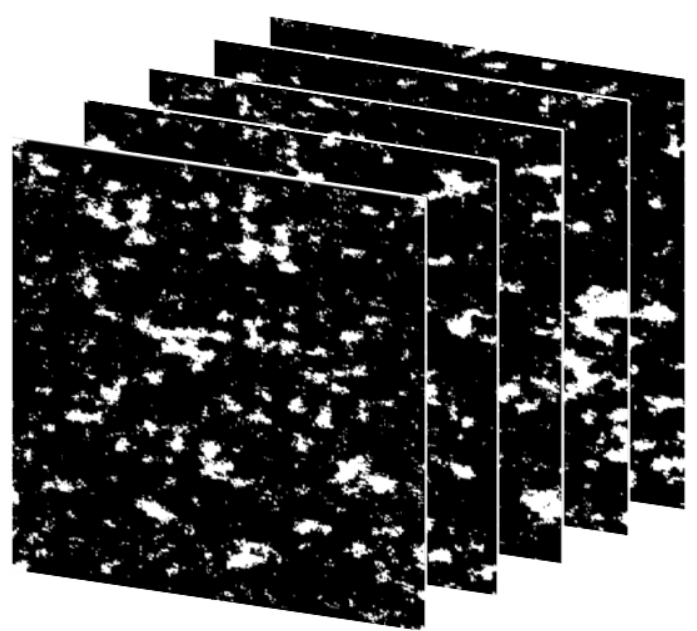

(b)

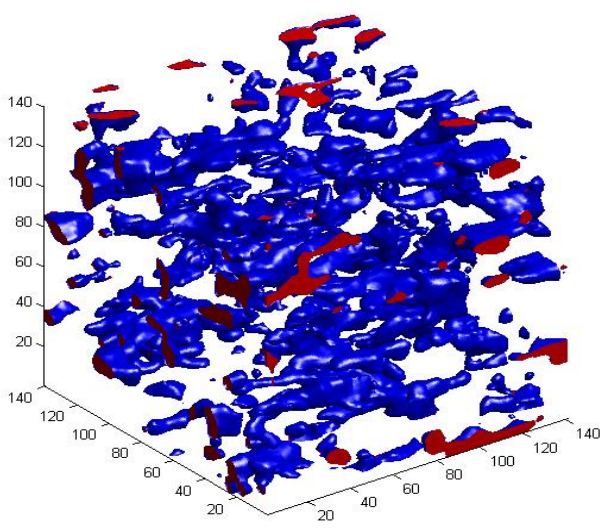

(d)

Fig. 8. (a) Original section used for 3-D reconstruction [13]. (b) Cross section of Reconstructed 3-D microstructure of HNT polypropylene composite based on the presented methodology. (c) 3-D reconstruction of the microstructure based on serial sectioning (FIB-SEM) [13] . (d) 3-D reconstruction of the microstructure based on the presented methodology.

\subsection{Error Analysis}

Presented reconstruction algorithm uses limited information and approximation schemes to operate. For this procedure two critical questions may arise: Using limited information about TPCFs, to what level of detail the real microstructure can be reconstructed? And is the reconstructed microstructure unique? To answer these questions, we investigate possible sources of error. For the presented reconstruction algorithm, two sources of error are existing. Error in the approximation of 3-point correlation functions from TPCFs using Eq. (14), and possible error in the reconstruction of microstructure by phase recovery algorithms. For every vector, the approximation error is defined by 
Pre-print of: Ali Hasanabadi, Majid Baniassadi, Karen Abrinia, Masoud Safdari \& Hamid Garmestani (2016). 3D microstructural reconstruction of heterogeneous materials from 2D cross sections: A modified phase-recovery algorithm. Computational Materials Science. DOI: 10.1016/j.commatsci.2015.09.015

$$
\text { Error }=\frac{\left|T P C F_{\text {exact }}-T P C F_{\text {approximated }}\right|}{T P C F_{\text {exact }}} .
$$

Baniassadi et al. [21] used Eq. (26) to analyze the approximation error for over 40,000 vectors with different magnitude and lengths, and they report an average error of $8 \%$. Using Eq. (26), the error extent was analyzed in the current study for the cases of two-dimensional approximation (section 4.1), 3D anisotropic material (section 4.3), and experimental verification (section 4.4) resulting an average error of $12 \%, 8 \%$ and $7 \%$, respectively.

It should be noted that accurate estimation of 3D full set TPCFs based on 2D cross sections with zero error intrinsically is not possible except for trivial cases. For example for isotropic material if two cross section are compared with each other, their statistical properties will be approximately the same but not identical. Accurate 3D full set TPCFs only achievable where related 3D microstructure be present.

For a given vector, the second source for error which originating from the phase recovery algorithms, is defined by

$$
\text { Error }=\frac{\left|T P C F_{\text {approximated }}-T P C F_{\text {reconstructed }}\right|}{T P C F_{\text {approximated }}} \text {. }
$$

Fig. 9 shows the convergence history plot for the presented reconstruction technique. Average error is calculated based on Eq. (27) for all iterations. It can be observed that with respect to this error measure, the reconstructed microstructure quickly converges to the solution for all examples presented in this study (below $10^{-7}$ after 100 iterations).

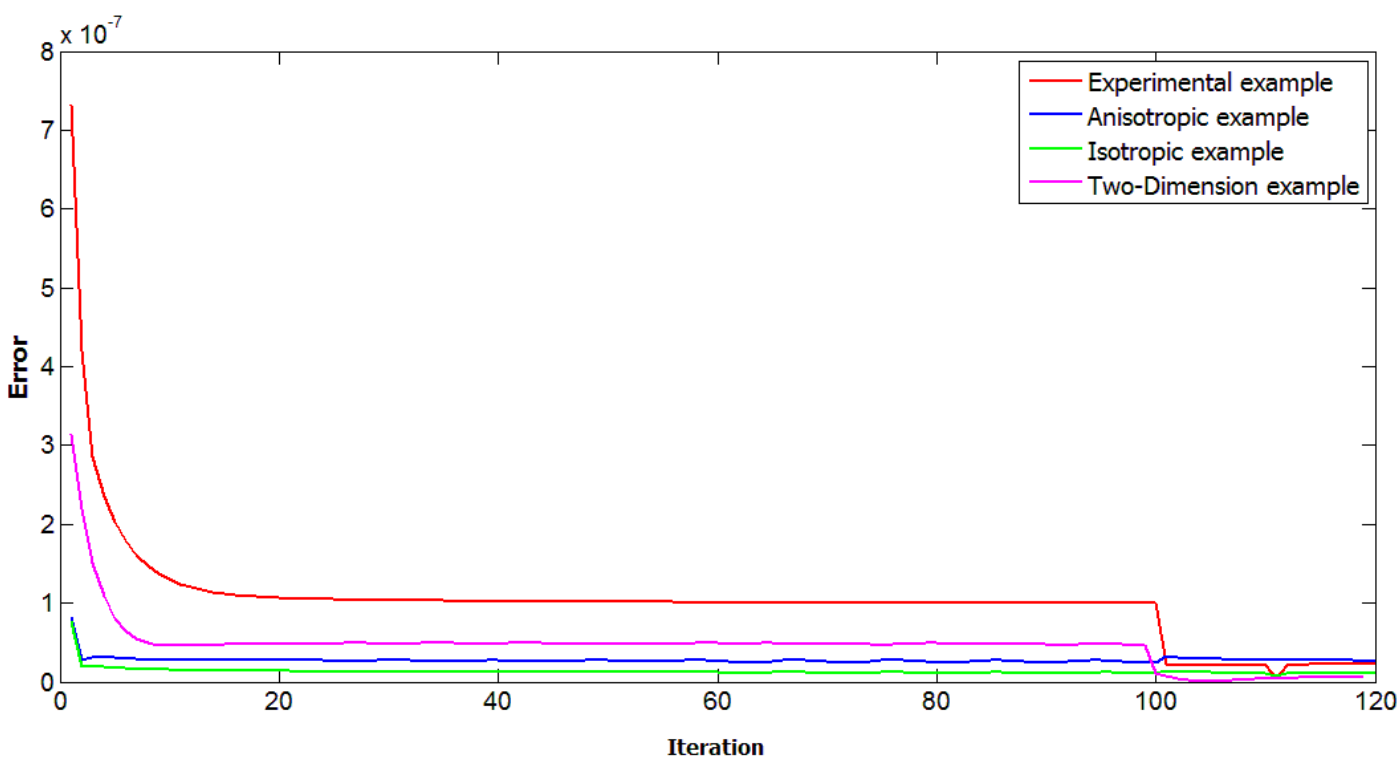

Fig. 9. History plot for error described in Eq. 27 for all examples of this paper.

About the convergence of algorithm it should be mentioned that calculated 3D full set TPCFs is an approximation of its accurate and physically realizable one. Because of these discrepancies, in reconstructing process using phase recovery method the reconstructed microstructure has TPCFs that are nearest to the approximated one and are also physically realizable. It is clear that the error between this third TPCFs set and actual one depends on the error of approximation (Eq. (26)).

The uniqueness of the reconstructed microstructure is questioned for any reconstruction algorithm which uses limited statistical information as the input. To answer this question, we notice that for the presented the uniqueness is mainly 
Pre-print of: Ali Hasanabadi, Majid Baniassadi, Karen Abrinia, Masoud Safdari \& Hamid Garmestani (2016). 3D microstructural reconstruction of heterogeneous materials from 2D cross sections: A modified phase-recovery algorithm. Computational Materials Science. DOI: 10.1016/j.commatsci.2015.09.015

influenced by two stages of the algorithm: 1) the approximation of the correlation functions; and 2) the reconstruction of microstructure from approximated correlation functions based on phase recovery approach. For the second stage, a study shows that a reconstructed eigen microstructure based on phase recovery algorithm is unique up to a translation and an inversion [24]. In the first stage of algorithm, an approximation scheme based on Eq. (17) is used which is not influenced by state (eigen/non-eigen) of the microstructure. Therefore, the presented reconstruction algorithm in general should provide a unique solution for an eigen microstructure.

\section{Conclusions}

In the present study, a new formulation was proposed to obtain a relation between the 3D full set and limited 2D correlation functions of heterogeneous materials. TPCFs of one cross section for isotropic materials and two perpendicular cross sections of anisotropic material were used in estimating 3D full set TPCFs. The approximation was developed using the conditional probability theory that is valid for multiphase heterogeneous materials. Reconstruction of microstructures were carried out using phase recovery algorithms. Comparison between the twopoint correlation functions obtained from the original 2D-reconstructed microstructure and the approximate correlation functions showed satisfactory agreement. A number of 3D reconstructions were performed for both isotropic and anisotropic microstructures, and it was shown that the proposed approximation scheme is very capable and well-suited for applications in which estimated correlation functions acquired from 2D cross sections of the microstructure are only available data for the reconstruction.

\section{Acknowledgments}

Authors gratefully acknowledge Dr.David T. Fullwood who provided detailed insight and great expertise for this research.

\section{References}

[1] S. Torquato, Random heterogeneous materials: microstructure and macroscopic properties. New York: Springer-Verlag, 2002.

[2] S. Torquato and G. Stell, "Microstructure of Two-Phase Random Media. I. The n-Point Probability Functions," Journal of Chemical Physics, vol. 77, pp. 2071-2077, 1982.

[3] S. Torquato and G. Stell, "Microstructure of Two-Phase Random Media. II. The Mayer-Montroll and Kirkwood-Salsburg Hierarchies," Journal of Chemical Physics, vol. 78, pp. 3262-3272, 1983.

[4] G. SAHELI, H. GARMESTANI, and B. L. ADAMS, "Microstructure design of a two phase composite using two-point correlation functions," Int J Comput Aid Des vol. 11, pp. 103-115, 2004.

[5] M. J. Beran, Statistical Continuum Theories, Monographs in Statistical Physics and Thermodynamics. New York: Interscience, 1968.

[6] M. Safdari, M. Baniassadi, H. Garmestani, and S. A.-H. Marvan, "A modified strong-contrast expansion for estimating the effective thermal conductivity of multiphase heterogeneous materials," JOURNAL OF APPLIED PHYSICS, vol. 112, p. 114318, 2012.

[7] B. L. Adams, S. R. Kalidindi, and Fulwood, David T., Microstructure-Sensitive Design for Performance Optimization. Waltham, MA 02451: Butterworth-Heinemann, 2013.

[8] N. Sheehan and S. Torquato, "Generating microstructures with specified correlation function," Journal of Applied Physics, vol. 89(1), pp. 53-60, 2001. 
Pre-print of: Ali Hasanabadi, Majid Baniassadi, Karen Abrinia, Masoud Safdari \& Hamid Garmestani (2016). 3D microstructural reconstruction of heterogeneous materials from 2D cross sections: A modified phase-recovery algorithm. Computational Materials Science. DOI: 10.1016/j.commatsci.2015.09.015

[9] B. L. Adams, X. Gao, and S. R. Kalidindi, "Finite approximations to the second-order properties closure in single phase polycrystals," Acta Materialia, vol. 53, pp. 3563-3577, 8// 2005.

[10] S. Torquato, "Effective stiffness tensor of composite media - I. Exact series expansion," Journal of the Mechanics and Physics of Solids, vol. 45, pp. 1421-1448, 1997.

[11] P. B. Corson, "Correlation functions for predicting properties of heterogeneous materials. I. Experimental measurement of spatial correlation functions in multiphase solids," JOURNAL OF APPLIED PHYSICS, vol. 45, pp. 3159-3164, 1974.

[12] M. S. Talukdar, O. Torsaeter, M. A. Ioannidis, and J. J. Howard, "Stochastic reconstruction, 3D characterization and network modeling of chalk," J. Petrol. Sci. Eng. , vol. 35, pp. 1-21, 2002.

[13] A. Sheidaei, M. Baniassadi, M. Banu, P. Askeland, M. Pahlavanpour, N. Kuuttila, et al., "3-D microstructure reconstruction of polymer nano-composite using FIB-SEM and statistical correlation function," Composites Science and Technology, vol. 80, pp. 47-54, 2013.

[14] J. A. QUIBLIER, "A New Three-Dimensional Modeling Technique for Studying Porous Media," Journal of Colloid and Interface Science, vol. 98, pp. 84-102, 1984.

[15] P. M. Adler, C. G. Jacquin, and J. A. QUIBLIER, "FLOW IN SIMULATED POROUS MEDIA," Int. J. Multiphase Flow, vol. 16, pp. 691-712, 1990.

[16] A. Lanzini, P. Leone, and P. Asinari, "Microstructural characterization of solid oxide fuel cell electrodes by image analysis technique," Journal of Power Sources, vol. 194, pp. 408-422, 2009.

[17] C. L. Y. Yeong and S. Torquato, "Reconstructing random media II. Three-dimensional media from two-dimensional cuts," PHYSICAL REVIEW E, vol. 58, pp. 224-233, 1998.

[18] B. Bochenek and R. Pyrz, "Reconstruction of random microstructures-a stochastic optimization problem," Computational Materials Science, vol. 31, pp. 93-112, 9// 2004.

[19] V. Sundararaghavan and N. Zabaras, "Classification and reconstruction of three-dimensional microstructures using support vector machines," Computational Materials Science vol. 32, pp. 223-239, 2005.

[20] M. Baniassadi, H. Garmestani, D. S. Li, S. Ahzi, M. Khaleel, and X. Sun, "Three-phase solid oxide fuel cell anode microstructure realization using two-point correlation functions," Acta Materialia, vol. 59, pp. 30-43, 2011.

[21] M. Baniassadi, S. Ahzi, H. Garmestani, D. Ruch, and Y. Remond, "New approximate solution for Npoint correlation functions for heterogeneous materials " Journal of the Mechanics and Physics of Solids, vol. 60, pp. 104-119, 2012.

[22] M. Baniassadi, M. Safdari, H. Garmestani, S. Ahzi, P. H. Geubelle, and Y. Remond, "An optimum approximation of n-point correlation functions of random heterogeneous material systems," THE JOURNAL OF CHEMICAL PHYSICS vol. 140, p. 074905, 2014.

[23] R. W. Gerchberg and W. O. Saxton, "A practical Algorithm for the Determination of Phase from Image and Diffraction Plane Pictures," OPTIK, vol. 35, pp. 237-246, 1972.

[24] D. T. Fullwood, S. R. Niezgoda, and S. R. Kalidindi, "Microstructure reconstructions from 2-point statistics using phase recovery algorithms," Acta Materialia, vol. 52, pp. 942-948, 2008.

[25] D. T. Fullwood, S. R. Niezgoda, B. L. Adams, and S. R. Kalidindi, "Microstructure sensitive design for performance optimization," Progress in Materials Science, vol. 55, pp. 477-562, 8// 2010.

[26] S. Torquato, "Exact conditions on physically realizable correlation functions of random media " $J$. Chem. Phys., vol. 111, pp. 8832-8837, 1999.

[27] A. M. Gokhale, A. Tewari, and H. Garmestani, "Constraints on microstructural two-point correlation functions," Scripta Materialia, vol. 53, pp. 989-993, 2005.

[28] T. T. SOONG, FUNDAMENTALS OF PROBABILITY AND STATISTICS FOR ENGINEERING West Sussex: John Wiley \& Sons Ltd, 2004. 
Pre-print of: Ali Hasanabadi, Majid Baniassadi, Karen Abrinia, Masoud Safdari \& Hamid Garmestani (2016). 3D microstructural reconstruction of heterogeneous materials from 2D cross sections: A modified phase-recovery algorithm. Computational Materials Science. DOI: 10.1016/j.commatsci.2015.09.015

[29] D. T. Fullwood, S. R. Kalidindi, S. R. Niezgoda, A. Fast, and N. Hampson, "Gradient-based microstructure reconstructions from distributions using fast Fourier transforms," Materials Science and Engineering A, vol. 494, pp. 68-72, 2008.

[30] Y. Jiao, F. H. Stillinger, and S. Torquato, "Modeling heterogeneous materials via two-point correlation functions: Basic principles," PHYSICAL REVIEW E, vol. 76, p. 031110, 2007.

[31] Y. Jiao, F. H. Stillinger, and S. Torquato, "Modelling Heterogeneous materials via two-point correlation functions. II. Algorithmic details and applications," PHYSICAL REVIEW E, vol. 77, p. 031135, 2008.

[32] S. R. Singiresu, Engineering Optimization Theory and Practice. New Jersey: John Wiley \& Sons, 2009. 